Ronald N. Kostoff, Raymond G. K oytcheff and Clifford G.Y . Lau

\title{
The growth of nanotechnology literature
}

ABSTRACT. Global nanotechnology research article production has exhibited exponential growth for more than a decade. The most rapid growth over that time period has come from East Asian nations, notably China and South Korea. Some of this apparent rapid growth (in China for example) is partially due to a non-negligible fraction of domestic low impact factor journals recently being accessed by the SCI/SSCI, rather than by increased sponsorship or productivity. While the U.S. remains the leader in aggregate nanotechnology research article production, in some selected nanotechnology sub-areas China has achieved parity or taken the lead. The main institution co-publishing groups are East Asian: one each from China, Japan, and South Korea. Publication connectivity among institutions is much weaker than common interest or citation connectivity. Correlation of institutions by the journals they cite reveals four nationality-based (or locality-based) clusters: Chinese, Japanese, American, and European. Institutions from the same nationality group cite the same focused journals (primarily, but not exclusively, domestic). Correlation of institutions by documents they cite reveals that only the Chinese institutions constitute a strongly-connected network. The dominant country co-publishing network is a complex web of mainly European nations roughly following geographic lines: Nordic, Central Europe, Eastern Europe, and a Western Europe/Latin American group of Romance language nations. There is also a UK component country network, but it is not linked to the interconnected continental members of the European Union. Correlation of countries by common thematic interest shows two major poles: U.S. and China. The U.S. pole is strongly connected thematically to a densely connected network of English-speaking North American representatives, Western/Central European nations, and most of the East Asian allies. China is relatively isolated except for India, and the Eastern European and Latin American representatives are outside the main network as well. There is a clear distinction between the publication practices of the three most prolific Western nations and the three most prolific East Asian nations. The Western nations publish in journals with almost twice the weighted average impact factors of the East Asian nations. Much of the difference stems from the East Asian nations publishing a non-negligible amount in domestic low impact factor journals, while the Western nations publish in higher impact factor international journals. Additionally, some of the Asian countries (e.g. China) are publishing in journals whose initial access date in the SCI/SSCI is relatively recent. If China is publishing a non-negligible fraction of its research output in newly-accessed relatively low impact factor journals, then some of its apparently rapid growth will not be in the traditional sense of increased sponsorship or productivity, but rather due to the SCI/SSCI's decision to access existing journals' articles. From another perspective, China's research article production may have been somewhat more competitive for decades, but was artificially suppressed by many of its journals' non-inclusion in the SCI/SSCI until only recently. Of the thirty institutions publishing the most nanotechnology papers, four are from the U.S., whereas of the twenty-five institutions producing the most cited papers, twenty-one are in the U.S. The two journals that contain the most cited nanotechnology papers since 1991 are Science and Nature, and the two countries that lead in production of the most cited papers are the U.S. and Germany, with the U.S. having four times the number of most cited papers as Germany. The U.S. and Germany account for forty percent of the most cited papers, while the high paper volume production East Asian countries of China and South Korea account for two percent of the most cited papers. The retrieved records for 2005 can be divided into two main categories with similar numbers of records (first level). One category focuses on phenomena and thin films (32 983 records), and the other category focuses on materials/structures (31 742 records). The 'phenomena' component of the first category is roughly four times the size of the 'films' component, whereas the 'nanotubes' component of the materials/structures category is about one ninth the size of the non-nanotubes 
component. A six factor analysis shows that the six key applications themes include: tribology, electronic devices and circuits, electrochemcial energy storage/production, optical electronics, optical transmission, microelectronic systems. Except for the first factor (tribology), all the other factors are electronic devices and components. A seven factor analysis shows that the seven key health and environment themes include: antibodies/immunity, cancer therapy, connective structures, nervous system pathology, bacterial infections/environmental, viral infections, blood flow/purity. Most of the health effort focuses on acute and degenerative diseases, rather than orthopedics. The environmental effort appears minimal and disjointed.

Nanotechnology Perceptions 2 (2006) 229-247

Nonsubscribers: purchase individual article 